\title{
Estimating Damaged Volume of Historic Pagodas in Bagan after Earthquake using 3D Hough Transform
}

\author{
Thida Aung", Myint Myint Sein* \\ ${ }^{\#}$ Faculty of Computer Science, University of Computer Studies, Yangon, No. (4) Road, Shwe Pyi Thar, Yangon, 11411, Myanmar \\ E-mail: tdathida@ucsy.edu.com
}

*Geographic Information System Lab, University of Computer Studies, Yangon, No. (4) Road, Shwe Pyi Thar, Yangon, 11411, Myanmar E-mail:myint@ucsy.edu.mm

\begin{abstract}
On $24^{\text {th }}$ August 2016, the magnitude of a 6.8 earthquake struck in Bagan from the depth of 52 miles. This earthquake caused much damage in historic pagodas in Bagan, one of the archeological houses in Asia. Analyzing the affected areas is an essential task for the restoration and reconstruction of historic buildings after a disaster. Traditional methods of detecting damage to buildings focus on detecting 2D changes (i.e., only the appearance of the image), but the 2D information provided by the image is not sufficient when it involves detecting damage to buildings is often not precise. For finding out the solution, a method of 3D change detection is needed for estimating the volumes of damaged pagodas after the earthquake. The proposed system aims at producing a quick assessment of the damaged pagodas accurately and correctly. This system estimates the damaged volume of the pagoda based on the nature of the 3D point clouds. Post-earthquake photos are taken using an anonymous aircraft (UAV) and point cloud data is generated using VisualSFM software. The 3D Hough transform is used to find the intersection of the tower vertex and the 3D vertex at the line boundary. Besides, the proposed system can detect the reformed structure of the entire pagoda. The results show that the proposed approach facilitates the automated 3D detection of damaged pagodas and is a time-saving method for estimating the volume of damage caused to precious historic pagodas after a disaster.
\end{abstract}

Keywords - 2D/3D change detection; point clouds; Unnamed Aerial Vehicle (UAV); VisualSFM; 3D hough transform.

\section{INTRODUCTION}

Cultural heritage is not only a valuable monument for the generations but also an important thing to renovate the glory of the past of the country. It is the essential thing that reveals the many aspects of religion, traditions, and beliefs. Cultural heritage is one category of the heritages that refers to cultural aspects like monuments and historic sites. Detailed damage assessment for cultural heritage after the calamity has gotten a necessary assignment in compelling crisis reaction and recuperation. In crisis reaction and recuperation, a manual appraisal is costly and tedious. Sometimes the urgent response is impossible in response. Remote sensing and geographic information system (GIS) is a valuable elective route for the harm evaluation process. We can get the satellite and aerial images effectively (UAV images) after the disaster occurred. We can get a critical harm evaluation utilizing the brushing the GIS advances and computerized picture handling. These days, in digital image processing, numerous procedures are utilized to identify harms brought about by natural disasters such as landslide, earthquake, and fire and flood. There are commonly two sorts of identifying the harms; object-based harmed identification and locale-based harmed discovery. Article based harmed recognition accentuates the shape, textures, background information and spectral information of the particular picture.

Traditional change discovery techniques were primarily created on radiometric data investigation of multi-worldly remote detected unearthly or optical pictures. Numerous applications, for example, checking land use/land cover classes, calamity evaluation, are created by utilizing change discovery methods. Change recognition results utilizing just 2D image's data are frequently affected by a lot of bogus alerts; for the most part, brought about via occasional varieties, diverse climate conditions, shadows, and impediments. Conventional structure harm change identification is primarily centered around 2D change systems, mainly dependent on the presence of the image. In $2 \mathrm{D}$ change, the method can figure the changed and unaltered territory of the structure; however, it is inadequate and exacts the entire structure change identification. So the change of the structure harm is wanted dependent on the $3 \mathrm{D}$ 
includes on 3D point mists acquired from aeronautical pictures. Because of the similar spectral characteristics, it is hard to recognize structures from other artificial developments like bridges and streets. Besides, 2D image's data-based techniques cannot be effectively used to decide the progressions at an individual structure level and investigate the volumetric data changes. Along these lines, inquire about change discovery on $3 \mathrm{D}$ point mists stays a functioning theme and new systems are requested to viably utilize accessible information from satellite, airborne, even low elevation platforms [1].

There are motivations why the proposed system emphasizes on estimating the damaged volume of $3 \mathrm{D}$ historic pagodas in Bagan. After the earthquake, the buildings and the pagoda were cracked or damaged. However, the whole building or pagoda is not damaged; only the part of the building is partially damaged. So, it is not quite disappeared from the earth. The first motivation is that some pagoda is partially destroyed, and it is not disappeared from the earth's surface. So, the damaged region of the pagoda does not easily calculate from this image. In this case, the traditional 2D Changed Detection technique cannot be used.

Another motivation is that in traditional 2D change detection, the damaged region of the building or pagoda is calculated from one side of the image. The damaged region from another side cannot be seen and estimated. Therefore, this change detection technique cannot show accurate and correct results. 3D Change detection should be applied in this case. Many papers and techniques are proposed to detect the damaged portion of the whole building. Most of them are based on the geometric mathematics especially intersecting points and vanishing parallel lines of the 3D building.

A method of calculating the volume of a 3D point cloud based on the slice method. Point clouds are generated by a point cloud laser data acquisition system. First, a projection plane is determined, and the projection plane is placed as a XOY plane, and finally, a three-dimensional point clouds are cut out in the $\mathrm{Z}$-axis direction along with the projection coordinate system [2]. A method of estimating the volume of an object is based on a 3D point cloud. First, cut the 3D point cloud along the z-axis into pieces of equal thickness. Divide each slice along the $y$-axis and divide each slice into subintervals along the $\mathrm{x}$-axis. By using the minimum and maximum coordinates in each subsection, two functions can be configured for the area in each half-section. Then, the area of each slice is estimated by combining the curves and integrated along the $\mathrm{z}$-axis of the estimated volume of the object [3].

A. Victor described the estimation of volume in a point cloud using a multi-agent system. We can take up this problem again and calculate the cell size of the Voronoi point cloud map. Estimate the cell size using the Monte Carlo method and distribute the workload to the agents. Later, we identified promising clients for future work clustering [4].

\section{THE MATERIAL AND METHODS}

The purpose of this system is to introduce the automatic $3 \mathrm{D}$ change detection on the point clouds. This section explains the combining strategies to get the damaged volume of the specific pagoda.

\section{A. Study Area and Data Collection}

Bagan, the ancient city, the archeological house of Myanmar, is the study area (see Fig. 1) where occurred magnitude of 6.8 earthquakes on August 24, 2016. Bagan is in the Mandalay Region of Myanmar. Founded between the 11 th and 13th centuries. There are over 10,000 Buddhist temples, stupas and monasteries.

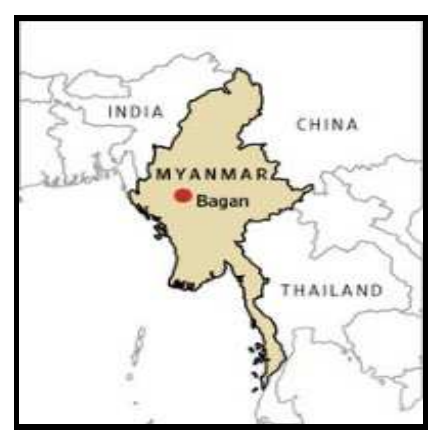

Fig. 1 Location of Bagan

Today there are more than 2,200 temples and pagodas. After striking the earthquake, about 397 pagodas are damaged and among them, 15 pagodas have huge damaged. GIS Lab performed the data collection for taking the aerial photos of damaged pagodas from The University of Computer Studies, Yangon. The UAV system used in the proposed system was a VTOL (vertical take-off and landing) hexacopter designed and manufactured by Sea Air Land (SAL) Engineering, equipped with a Canon EOS 550D calibrated digital camera. There are approximately 300 aerial photographs of a particularly damaged pagoda, each with a resolution of $72 \mathrm{dpi}$ [5], [6].

\section{B. General Workflow of Proposed System}

The general workflow of the proposed system is as shown in following figure 2 .

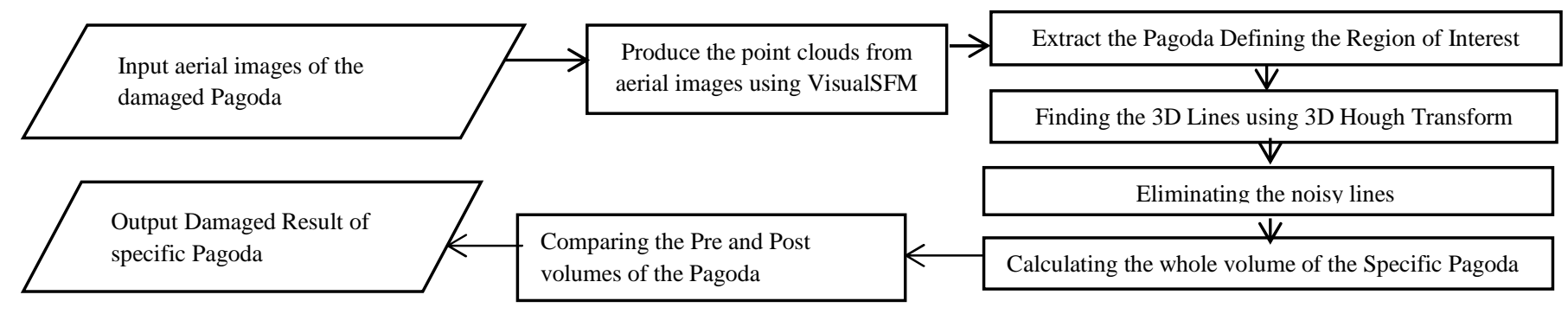

Fig. 2 Workflow of Proposed System 


\section{Producing 3D Point Clouds using VisualSfM Software}

The best method in open source software for 3D reconstruction would be Structure-from-Motion (SFM) based methods. The challenges with flying platforms would be vibrations and video stability, but techniques exist to deal with these to a great extent. Visual SFM, by default, would generate relatively sparse point clouds but with surface fitting and robust priors-based reconstruction that can be improved the results considerably. Structure from motion (SFM) is a technique that can be used for obtaining topographic data from digital imagery that is widely used in the geographic information system. We have a few software options for processing photographs to obtain 3D point clouds containing XYZ coordinates and RGB color index.

There is freely available SfM software, including Bundler Photogrammetry Package (Snavely et al. 2006), SFMToolkit, PhotoSynth Toolkit, Python Photogrammetry Toolbox, VisualSFM (Wu 2013), and 3DF Samantha. Proprietary software includes Agisoft PhotoScan, Acute3D, PhotoModeler, and 3DF Zephyr. Web-based services include Photosynth, Arc3D (Tingdahl and Van Gool 2011), CMP SfM Web Service, and Autodesk 123D Catch. Chang chang $\mathrm{Wu}$ created VisualSFM and we guess more information about VisualSFM on Dr. Wu's website (http://ccwu.me/vsfm). The general workflow of visual SFM (see Fig. 3) is as follows:

- Importing Images

- Matching Images

- Creating the Sparse Reconstruction

- Creating Dense Reconstruction [7]

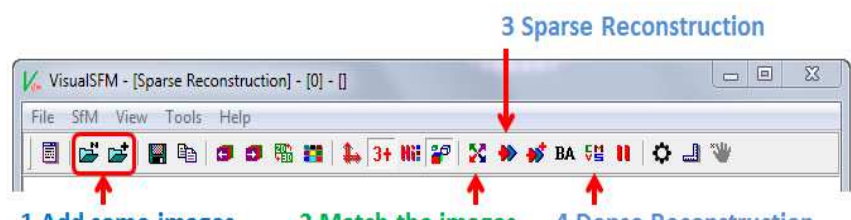

1 Add some images 2 Match the images 4 Dense Reconstruction

Fig. 3 Workflow of VisualSFM Software

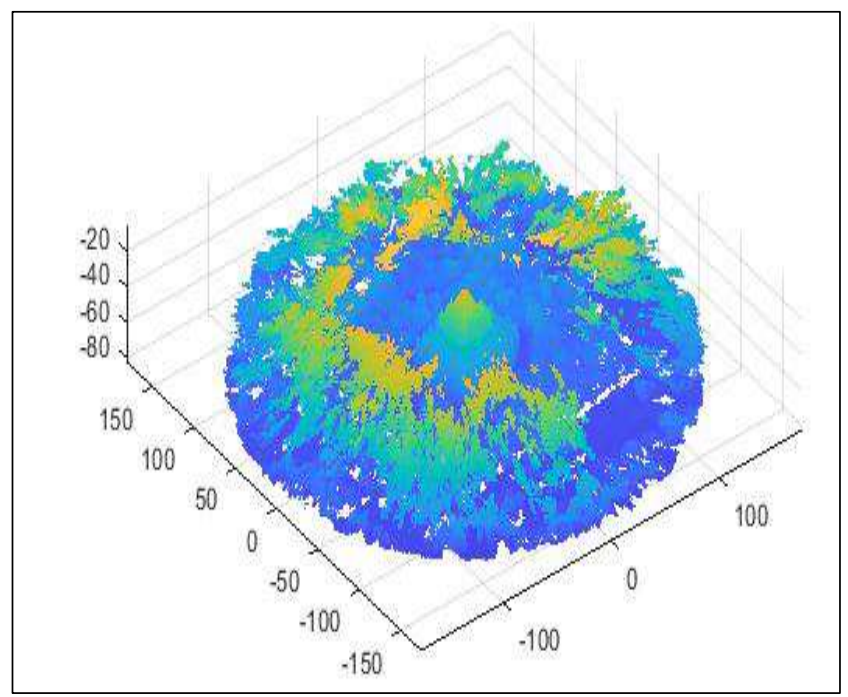

Fig. 4 Output point clouds of Sulamani Pagoda in Bagan

\section{Defining the ROI of Pagoda}

That point cloud datasets contain varying point densities. Therefore, the sparse outliers deduce the system's result even more. Some of the outlier noise can be solved by using statistical analysis on each 3D point cloud's neighborhood and dropping out the unnecessary points cloud. The unnecessary points can be calculated on the computation of the distribution of points in the input dataset. The outliers are trimmed based on defining the region of interest (ROI). The noise point clouds can be reduced by doing this stage. ROI can be defined by clicking the four corners of the specific pagoda [8]. After eliminating the unnecessary point clouds, the output from the extracting boundary of Sulamani pagoda is shown in figure 5 .

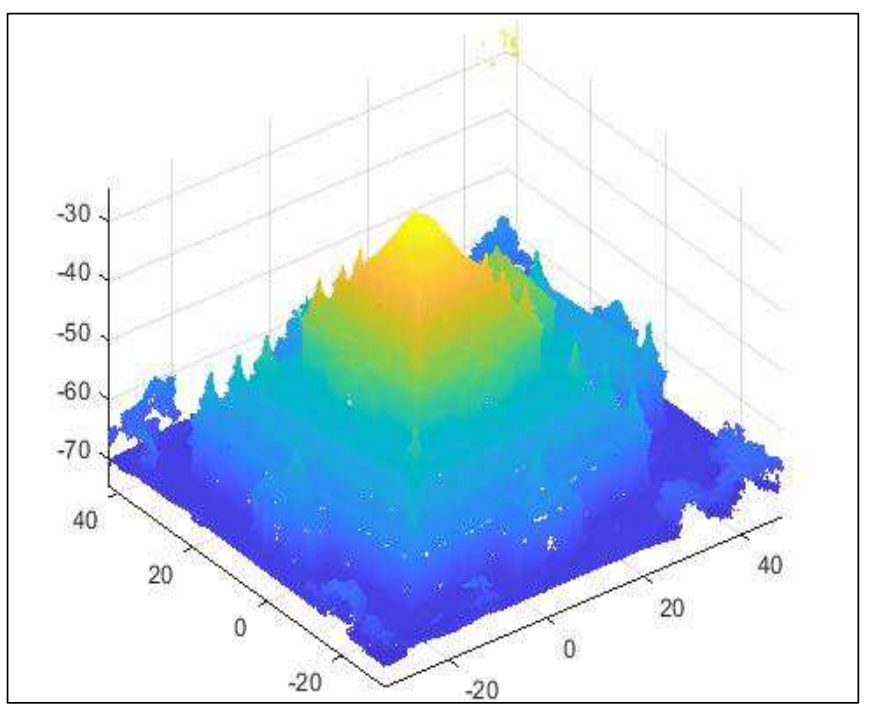

Fig. 5 Point Clouds of Sulamani Pagoda after defining ROI

\section{E. Detecting 3D Lines using 3D Hough Transform}

Hough (1962) implemented the Hough Transform for the detection of parametrized objects, mainly for lines and circles in digitalized images. Now, 3D Hough Transform focuses on the detection of the lines in 3D point clouds of the 3D historic Bagan Pagodas. The original Hough transform is operated on the $2 \mathrm{D}$ images. The extended $3 \mathrm{D}$ Hough transform contributes to the three-dimensional point clouds. The point clouds are given to the 3D Hough transform as the input to find all possible straight lines passing through the points. Each point has been voting for all lines in the parameter space [9].

The direction of the line can be calculated from $\theta$ for altitude (elevation) and $\phi$ for horizontal orientation (azimuth) (see figure 6 and 7) as shown in Equation 1. To overcome the aniti-parallel directional vectors for same line let the restrictions of angle ranges for $\theta$ and $\phi$ be $0 \leq \theta \leq \pi / 2$ and $-\pi$ $\leq \phi \leq \pi[10]$. For eliminating the redundancy through anitiparallel vector pairs in the $(x, y)$-plane $(b z=0)$ is removed with by $\geq 0$ if $b z=0$ and $b x=1$ if $b y=b z=0$.

$$
\vec{b}=\left(\begin{array}{c}
b_{x} \\
b_{y} \\
b_{x}
\end{array}\right)=\left(\begin{array}{c}
\cos \phi \cos \theta \\
\sin \phi \cos \theta \\
\sin \theta
\end{array}\right)
$$



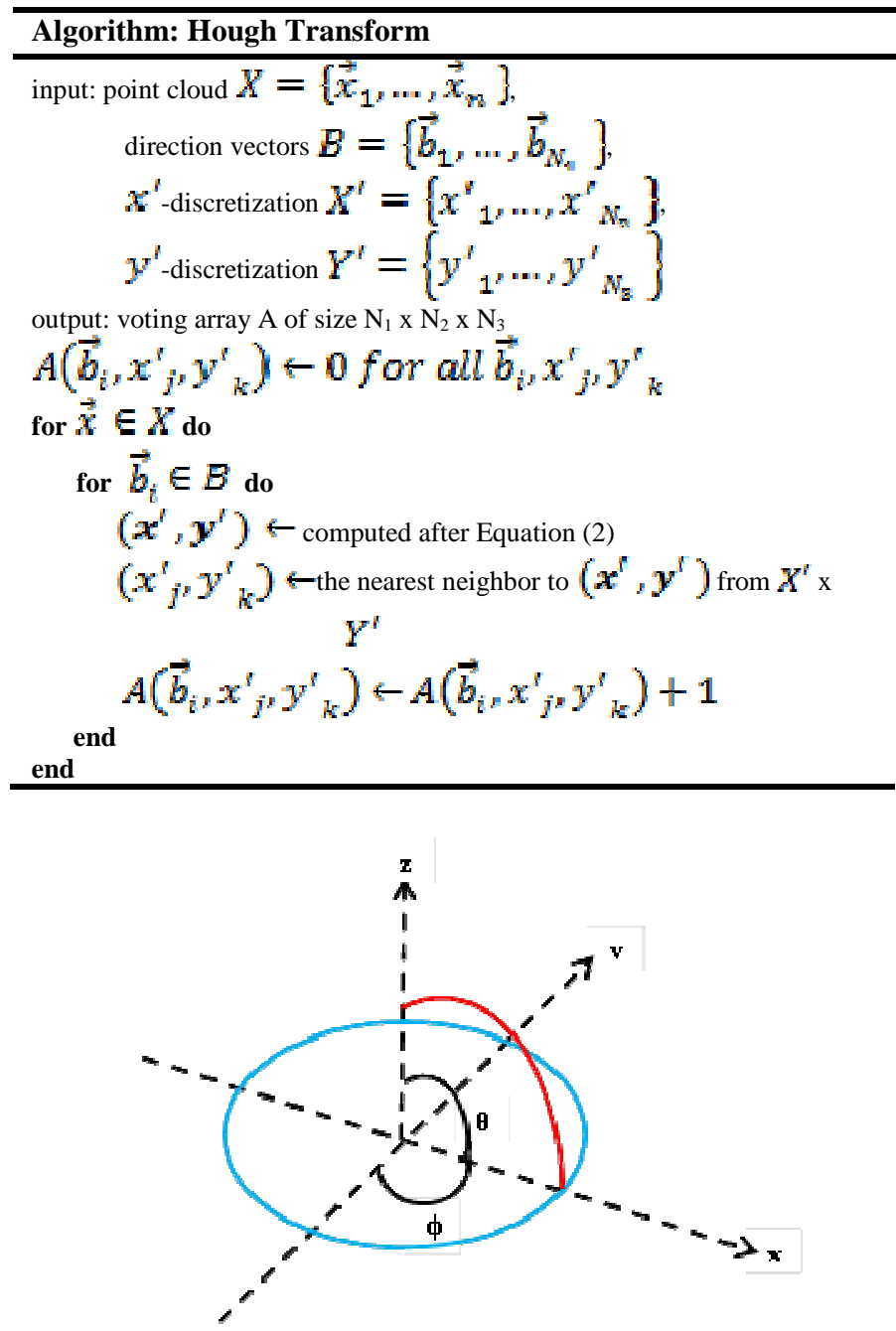

Fig. $6 \phi$ and $\theta$ as azimuth (blue) and elevation (red)

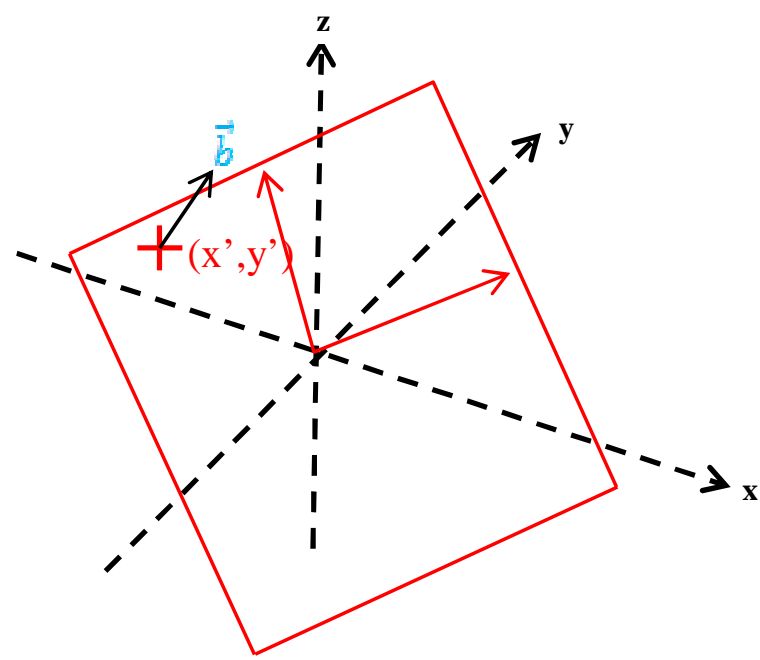

Fig. 7 Line parametrization by Roberts with $x^{l}, y^{l}$ (red) and the line's directional vector $\vec{b}$ (blue)

Roberts defined $x^{\prime}$ and $y^{\prime}$ as the coordinates of the intersection of the line and the plane in the context of $2 \mathrm{D}$ coordinates of the plane (see figure 7$)$. Let $\vec{p}=\left(\mathrm{p}_{\mathrm{x}}, \mathrm{p}_{\mathrm{y}}, \mathrm{p}_{\mathrm{z}}\right)$ on the line, the parameter $x^{\prime}$ and $y^{\prime}$ are obtained in equation 2 : $x^{\prime}=\left(1-\frac{b_{x}^{2}}{1+b_{z}}\right) p_{x}-\left(\frac{b_{x} b_{y}}{1+b_{z}}\right) p_{y}-b_{x} p_{z}$

$y^{\prime}=-\left(\frac{b_{z} b_{y}}{1+b_{z}}\right) p_{x}+\left(1-\frac{b_{y}{ }^{2}}{1+b_{z}}\right) p_{y}-b_{y} p_{y}$

A point $\vec{p}$ on the line can be obtained in the following equation (3):

$$
\vec{p}=x^{\prime} \cdot\left(\begin{array}{c}
1-\frac{b_{z} 2}{1+b_{z}} \\
-\frac{b_{x} b_{y}}{1+b_{z}} \\
-b_{x}
\end{array}\right)+y^{\prime}\left(\begin{array}{c}
-\frac{b_{x} b_{y}}{1+b_{z}} \\
1-\frac{b_{y}{ }^{2}}{1+b_{z}} \\
-b_{y}
\end{array}\right)
$$

\section{F. Cutting Top Conic Portion of Pagoda}

In Myanmar country, the ancient king builds the various types of pagodas. The types of towers can be distinguished by their shape and structure, such as Zedi, Pahto, and Phaya. The Buddha statue is known as Paya. Pafts are hollow square or rectangular buildings designed to resemble caves. Zedi is bell-shaped and generally strong. Figure 8 shows examples of images of Phaya, Pahto, and Zedi.

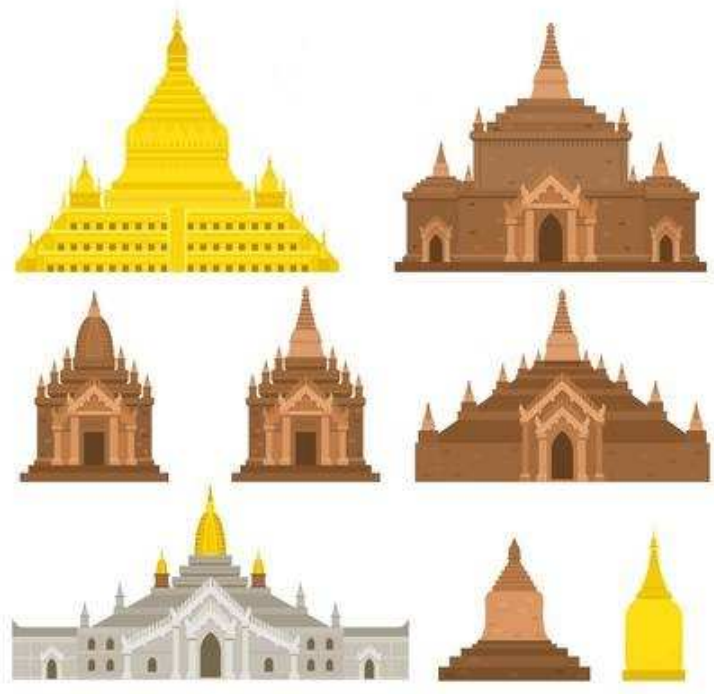

Fig. 8 Different Structures of Pagodas in Bagan

The Zedi vertices (Figures 9 and 10) are necessary to calculate to reconstruct the original shape. Now consider that the top intersection $(\mathrm{x}, \mathrm{y}, \mathrm{z})$ can be calculated as follows:

Let $l_{1}$ and $l_{2}$ is the two lines with vector forms;

$$
\mathrm{l}_{1}=\mathrm{Pt}_{1}+\mathrm{aVec}_{1}, \quad \mathrm{l}_{2}=\mathrm{Pt}_{2}+\mathrm{bVec}_{2}
$$

where $\mathrm{Vec}_{1}$ and $\mathrm{Vec}_{2}$ are the director vectors and $\mathrm{pp}_{1}$ point which belongs to $1_{1} ; \mathrm{Pt}_{2}$ point which belongs to $1_{2}$, respectively.

$\Leftrightarrow \mathrm{Pt}_{1}+\mathrm{aVec}_{1}=\mathrm{Pt}_{2}+\mathrm{bVec}_{2} \Rightarrow \mathrm{aVec}_{1}=\left(\mathrm{Pt}_{2}-\mathrm{Pt}_{1}\right)+$ $\mathrm{bVec}_{2} \mathrm{X} \mathrm{Vec}_{2}$ in the two sides with cross product:

$$
\mathrm{a}\left(\mathrm{Vec}_{1} \times \mathrm{Vec}_{2}\right)=\left(\mathrm{Pt}_{2}-\mathrm{Pt}_{1}\right) \times \mathrm{Vec}_{2}
$$

once "a" can be replaced it in the $1^{\text {st }}$ equation to get the point of intersection $(\mathrm{x}, \mathrm{y}, \mathrm{z})$. 
This equation is proof with the following calculation;

Take $\mathrm{Pt}_{1}=(1,0,0) \mathrm{Vec}_{1}=(2,3,1)$ and

$\mathrm{Pt}_{2}=(0,5,5) \mathrm{Vec}_{2}=(5,1,-3)$

$\mathrm{a}\left(\mathrm{Vec}_{1} \times \mathrm{Vec}_{2}\right)=\mathrm{a}(-10,-11,-13)$ and

26)

$$
\left(\mathrm{Pt}_{2}-\mathrm{Pt}_{1}\right) \times \mathrm{Vec}_{2}=(-1,5,5) \times(5,1,-3)=(-20,-22,-
$$

Since the latter is $2(-10,-11,-13)$, we have $a=2$, and the intersection point is

$\mathrm{Pt}_{1}+2 \mathrm{Vec}_{1}=(1,0,0)+(4,6,2)=(5,6,2)$

To find $\mathrm{b}$ and check the answer;

$\mathrm{Pt}_{2}+\mathrm{bVec}_{2}=(0,5,5)+\mathrm{b}(5,1,-3)$ which will equal $(5,6,2)$ when

$\mathrm{b}=1$.

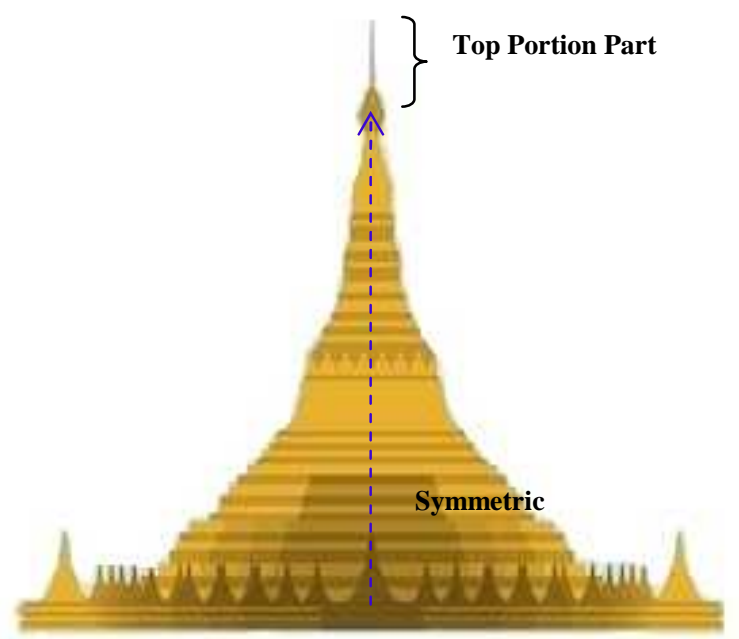

Fig. 9 Structures of Zedi

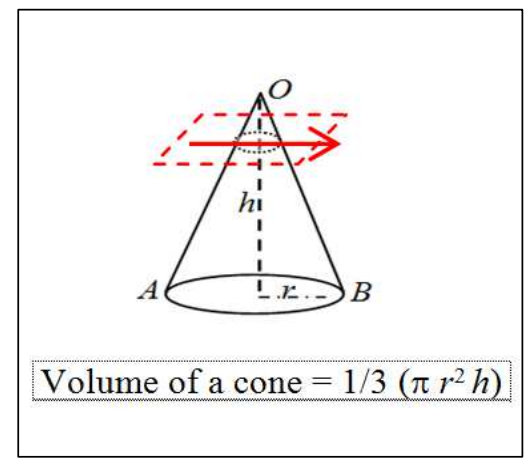

Fig. 10 Illustration of the Top Portion of Zedi

\section{RESULTS AND DISCUSSION}

For the proposed system, the system requirements are nearly i7-4790CPU with 3.6GHz, 32G RAM, 16G VGA. In implementing the proposed system, the main three stages can be observed. The first stage is taking the aerial photos of the damaged pagoda using the UAV (Hexacorpoter). The second phase is generating the $3 \mathrm{D}$ points clouds of the specific pagoda using the open-sourced software, visual SFM. The final part is calculating the volume of the damaged pagoda using 3D Hough Transform and cutting the top portion. In this section, the detail process and results are discussed systematically.

In the first stage, it takes about 45 minutes to take approximately 300 aerial photos with Canon EOS model $550 \mathrm{D}$ digital camera. The second stage generates the 3D point clouds of $10 \neg 106$ using visualSFM software. In the proposed system, the second stage takes a long time to generate the $3 \mathrm{D}$ points clouds. That is why the aerial photos of the damaged pagoda have a high resolution to get accurate point clouds. In the future, the resolution factor can be solved to reduce the running time. The extraction of ROI (Region-Of-Interest) is an important part of the final stage of the proposed system. After generating the 3D point clouds from visualSFM, there are many points clouds for the specific pagodas, including surround trees and buildings near the damaged pagoda. Therefore, the ROI of the pagoda is extracted to get the better performance of the proposed system. The following table (Table 2) shows the percentage of reducing point clouds of some pagodas after defying the ROI.

TABLE I

THE RESULT ON NO. OF POINT CLOUDS AFTER DEFINING ROI

\begin{tabular}{|c|l|c|c|c|}
\hline No. & \multicolumn{1}{|c|}{$\begin{array}{c}\text { Name of } \\
\text { Pagoda }\end{array}$} & $\begin{array}{c}\text { No. of } \\
\text { Original } \\
\text { Point } \\
\text { Clouds }\end{array}$ & $\begin{array}{c}\text { No. of Point } \\
\text { Clouds after } \\
\text { extracting } \\
\text { ROI }\end{array}$ & $\begin{array}{c}\text { Reduced } \\
\%\end{array}$ \\
\hline 1. & $\begin{array}{l}\text { A Naunt Phat } \\
\text { Late Pagoda }\end{array}$ & 7683845 & 2553410 & $66.77 \%$ \\
\hline 2. & $\begin{array}{l}\text { Ta Yoke Pye } \\
\text { Pagoda }\end{array}$ & 11528186 & 3153763 & $72.64 \%$ \\
\hline 3. & $\begin{array}{l}\text { Gu Pyaunt Gyi } \\
\text { Pagoda }\end{array}$ & 6662288 & 903107 & $86.45 \%$ \\
\hline 4. & $\begin{array}{l}\text { Zay Tee } \\
\text { Pagoda }\end{array}$ & 13124274 & 5270746 & $59.84 \%$ \\
\hline 5. & $\begin{array}{l}\text { Su La Ma Ni } \\
\text { Pagoda }\end{array}$ & 8279211 & 2993983 & $63.84 \%$ \\
\hline 6. & $\begin{array}{l}\text { Say Ta Nar Gyi } \\
\text { Pagoda }\end{array}$ & 20244339 & 6241256 & $69.17 \%$ \\
\hline 7. & $\begin{array}{l}\text { Ywar Haung } \\
\text { Gyi Pagoda }\end{array}$ & 9571758 & 1657174 & $82.69 \%$ \\
\hline
\end{tabular}

After extracting the required region of the pagoda, the unrequired part of noisy point clouds is remaining. Therefore, the noisy point clouds should be removed from the important region of the pagoda. The unrequired point clouds are eliminated using the point clouds clustering method on point clouds' location [12].

The final part of the proposed system is to estimate the volume; finding the straight lines among the $3 \mathrm{D}$ point clouds using the Hough Transform and cutting the top porting part of the specific pagoda. While finding the lines among the point clouds, sometimes some failure can become out. These are

- There is no enough memory for the 3D point clouds or Hough space accumulator array

- the point cloud contains many identical points

- it takes place overflow in an accumulator array counter [13].

The results of the 3D Hough Transform is the lines in the vector form with the following information;

$$
\text { npoints }=n, \mathrm{a}=\left(a_{x}, a_{y}, a_{z}\right), \mathrm{b}=\left(b_{x}, b_{y}, b_{z}\right)
$$

where $\mathrm{n}$ is the number of points that are lying on the same straight line, $\vec{a}=\left(a_{x}, a_{y}, a_{z}\right)$ is the anchor point that lies inline, 
and $\vec{b}=\left(b_{x}, b_{y}, b_{z}\right)$ is the line direction[14][15]. The detected lines are returned in decreasing order of the Hough space vote counts as in the following example:

npoints $=2970007, \mathrm{a}=(-13.796632,-13.791075,-13.785058), \mathrm{b}=$ $(0.577281,0.577351,0.577418)$

npoints $=4989, \mathrm{a}=(9.489355,4.441291,3.902953), \mathrm{b}=(0.590895,0.558838$, $0.581845)$

npoints $=4422, \mathrm{a}=(7.817226,10.019059,13.326892), \mathrm{b}=(0.566138$, $0.603609,0.561377)$

npoints $=3781, \mathrm{a}=(12.581729,13.519197,7.481504), \mathrm{b}=(0.564424$, $0.573193,0.594033)$

The straight lines through the point clouds of the damaged pagodas are shown in the result figures, respectively. The candidate straight lines should be needed to determine the top intersection points by eliminating the noisy lines. Zedi vertices are necessary to calculate to recreate the original shape [16]. After getting the upper intersection of the damaged pagoda, you can estimate the damaged volume. To calculate the percentage of the damaged region of the historical pagoda, firstly, width, height and length of the individual pagoda are estimated from the original reforming shape of the pagoda and collected from the ground truth data. Some geographic information is available on the historical database of the Ministry of Cultural and Religious Affairs, Myanmar. The damaged percentage is calculated on the number of filling pixels and the remaining pixels. Equation (4) is used to determine the damaged percentage of the historic pagodas. Figure 11-15 shows the detail results of each step of the proposed system and results on the damaged volume percentage [17], [18].

$$
\text { DamagedPer centage }=\left(\frac{\text { numberOfDa magedPixel } s}{\text { numberOfTo talPixels }}\right) \cdot 100 \%
$$

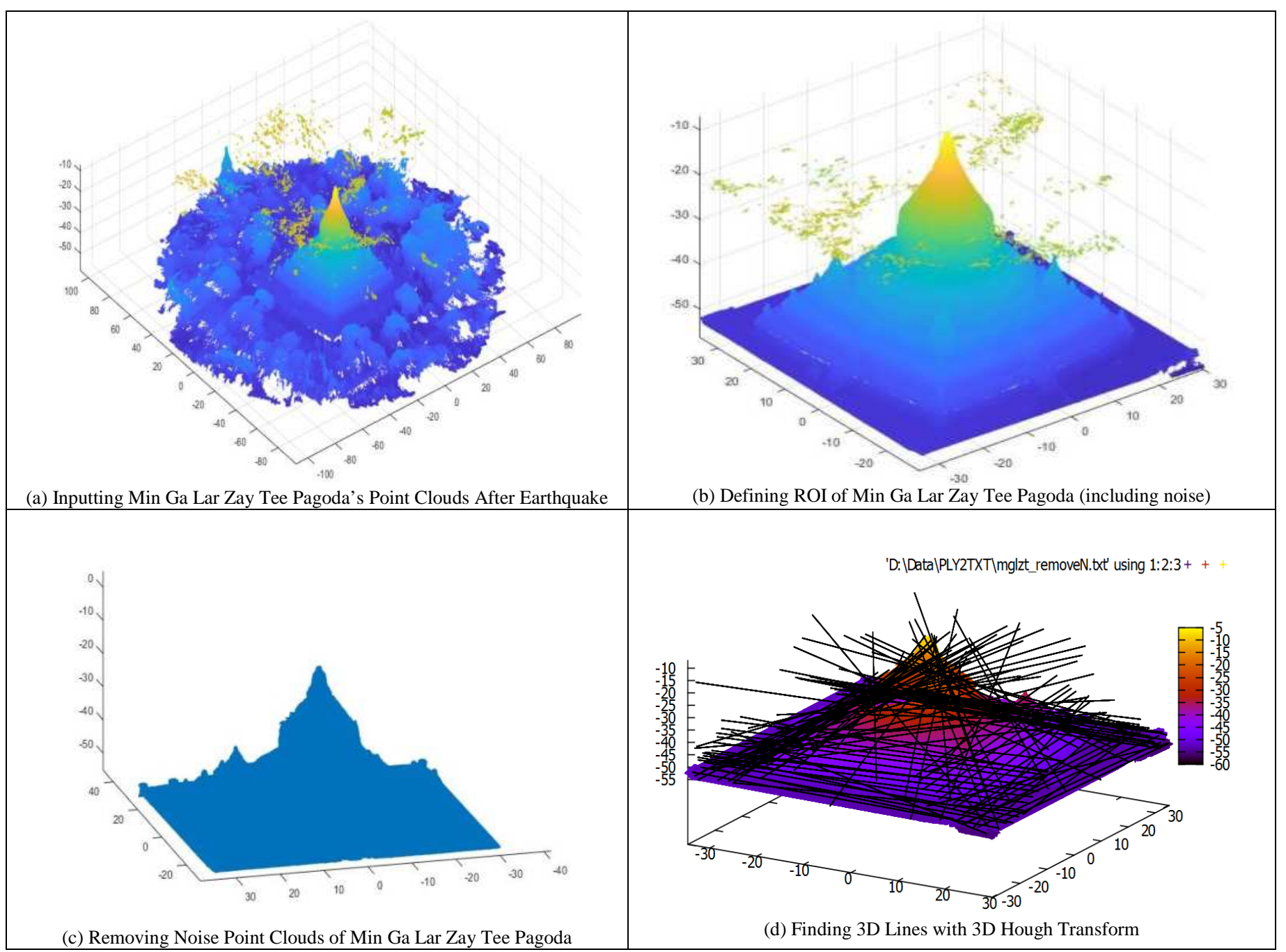

Fig. 11 Min Ga Lar Zay Tee Pagoda 


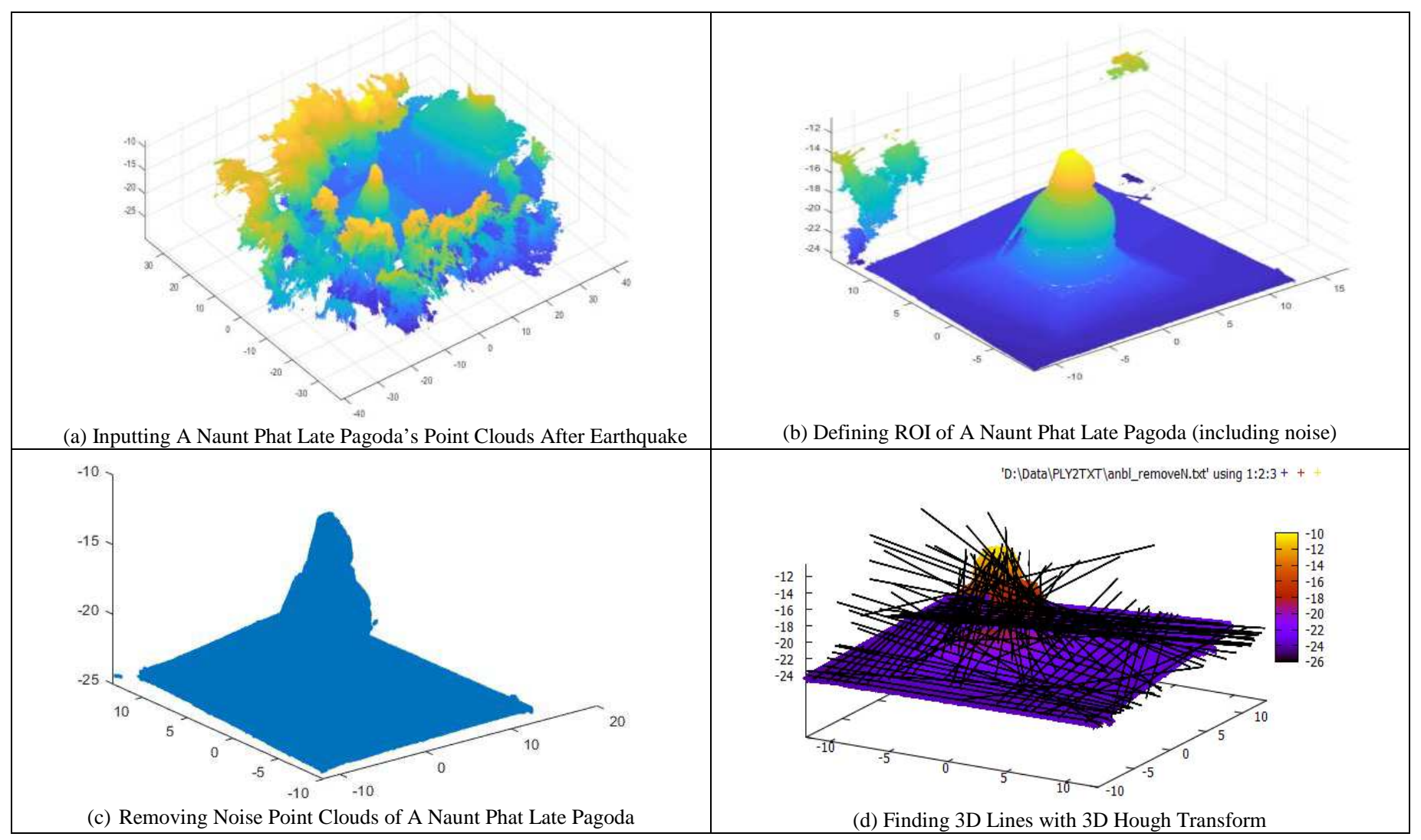

Fig. 12 Min Ga Lar Zay Tee Pagoda

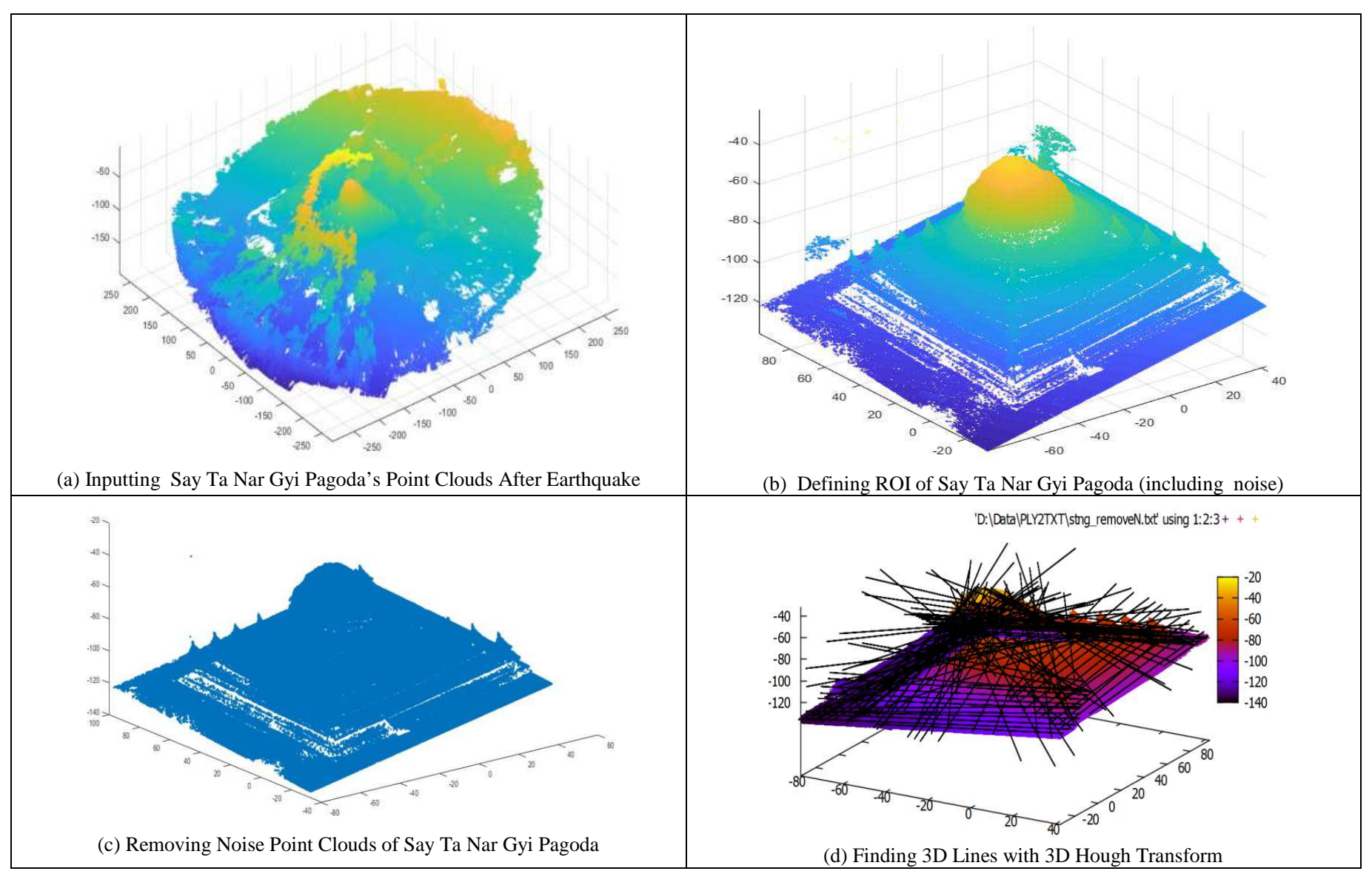

Fig. 13 Say Ta Nar Gyi Pagoda 


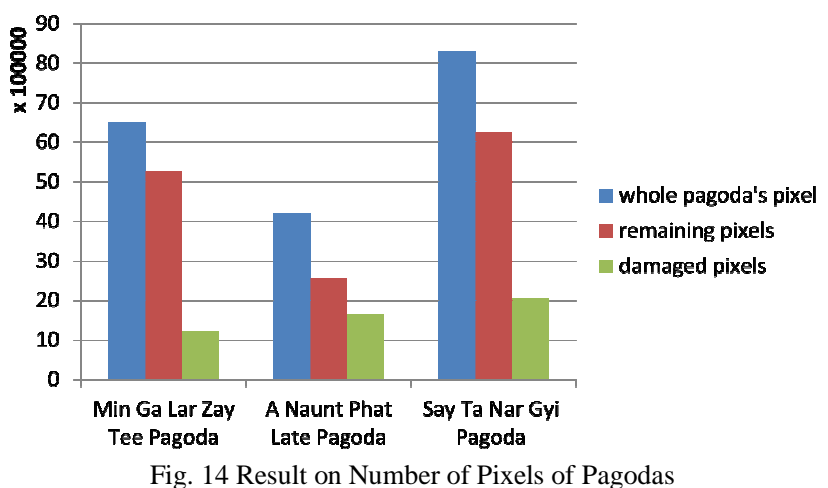

Fig. 14 Result on Number of Pixels of Pagodas

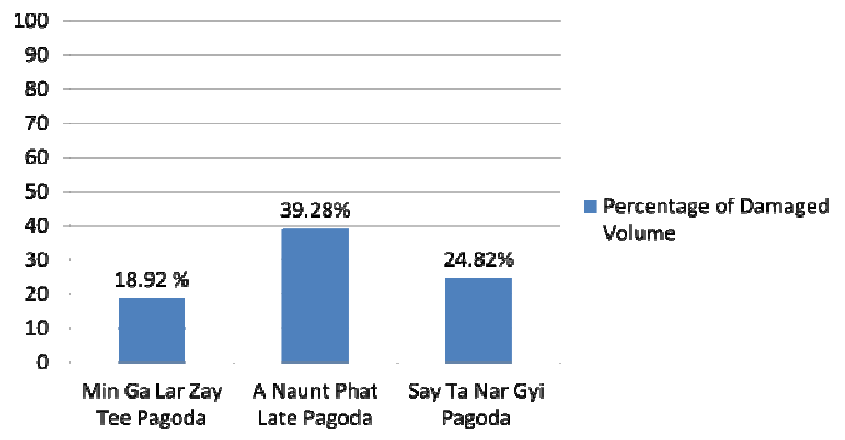

Fig. 15 Result on Percentage of Damaged Volume of Pagodas

\section{CONCLUSIONS}

The proposed method for estimating the damaged volume of the 3D historic pagoda is firstly implemented in Myanmar. Most of the estimated damaged volume is emphasized in 3D buildings. This proposed system is mainly contributed to detecting the damaged portion of pagoda based on the 3D structure of the historic pagoda after the earthquake. This study aims to preserve the cultural heritage of the old pagoda. The first part of this research work consists in restoring the original structure of the tower by taking damaged images using drones; the second part is producing the required point clouds using the open-source visualSFM software and the third part is estimating the damaged volume using 3D Hough transform and nature of the ancient pagodas' structures. Though the experimental results and discussion, it can be confirmed that the proposed method works adequately.

\section{ACKNOWLEDGMENT}

We would like to thank the Ministry of Religious Affairs and Culture, Myanmar for permitting me to take the postearthquake UAV photos in the Archaeological zone in Bagan. We would like to thank also National Institute of Information and Communications Technology (NICT), Japan for giving me to participate as one of the ASEANIVO Projects.

\section{REFERENCES}

[1] H. Shusong, D. Aixia, W. Xiaoqing, and W. Jinxia, "Earthquake-Induced Building Damage Detection Method Based on Normal Computation Of Neighboring Points Searching On 2d-Plane", IEEE International Geoscience \& Remote Sensing Symposium, pp. 4251-4254, 2016.

[2] Z. Yu, Z. Yikun, C. Hao, Y. Kaifeng and X. Hui, "A Method of 3D Point Cloud Volume Calculation Based on Slice Method", International Conference on Intelligent Control and Computer Application (ICCA), China, 2016.

[3] C. Wen-Chung, W. Chia-Hung, T. Ya-Hui and C. Wei-Yao, "Object Volume Estimation Based on 3D Point Clouds", Industrial Technology Research Institute, Taiwan, R.O.C, 2017.

[4] A. Victor, "Volume Estimation in Point Cloud Using Multi-Agent System", $19^{\text {th }}$ International Symposium on Symbolic and Numeric Algorithms for Scientific Computing (SYNASC), 2017

[5] A. Thida and S. Myint Myint, "Analyzing Damage region of 3D Historic Pagodas Images after the Earthquake", ICT Virtual Organization of ASEAN Institutes and NICT ASEAN IVO Forum, 2016.

[6] A. Thida and S. Myint Myint, H. Hiromitsu "Reconstruction of the Ancient Pagodas", IEEE $7^{\text {th }}$ Global Conference on Consumer Electronics (GCCE 2018), Nara, Japan, Oct 2018.

[7] (2013) W. Chang chang, "VisualSFM", [Online]. Available http://ccwu.me/vsfm

[8] Zuzan and R. Milan, "Object Volume Calculation from Large Noisy Point-Clouds", Czech Technical University in Prague, May, 2018.

[9] J. Manuel, D. Christoph and P. Regina,"Hough Parameter Space Regularisation for Line Detection in 3D", International Conference on Computer Vision Theory and Applications (VISAPP), pp. 345352, 2016.

[10] D. Christoph, S. Tilman and J. Manuel, "Iterative Hough Transform for Line Detection in 3D Point Clouds", Image Processing (2017) [Online], pp. 184 Available: https://doi.org/10.5201/ipol.2017.208

[11] C. Marco, V. Roberto and C. Rita, "3D Hough transform for sphere recognition on point clouds", Machine Vision and Applications, 25:1877-1891, 2014

[12] L. Xiaohu, L. Yahui and L. Kai, "Fast 3D Line Segment Detection from Unorganized", Computer Vision and Pattern Recognition, https://arxiv.org/abs/1901.02532,2019

[13] Z. Xin, M. John and K. Reinhard, "Volume Measurement Using a Laser Scanner", The International Archives of the Photogrammetry, Remote Sensing and Spatial Information Sciences, vol. XL-5, 2014.

[14] J. Ales, E. Miran, M. Igor, S. Ziga and S. Franc, "Volumetric models from 3D point clouds: The case study of sarcophagi cargo from a 2nd/3rd century AD Roman shipwreck near Sutivan on island Brac, Croatia", Journal of Archaeological Science, pp 143-152, 2015.

[15] W. Jinhu, G. Higinio, L. Roderik, A. Pedro and M. Massimo, "Automatic Estimation of Excavation Volume from Laser Mobile Mapping Data for Mountain Road Widening”, Remote Sens., 46294651, 2013.

[16] A. Bienert , C. Hess, H.-G. Maas and G. von Oheimb, "A VoxelBased Technique To Estimate The Volume Of Trees From Terrestrial Laser Scanner Data", The International Archives of the Photogrammetry, Remote Sensing and Spatial Information Sciences, vol. XL-5, ISPRS Technical Commission V Symposium, Riva del Garda, Italy, 2014.

[17] Z. Qijie, W. Yijing, L. Xianfa, X. Jiao and M. Qingxu, "A method of measuring stacked objects volume based on laser sensing", Measurement Science and Technology, Meas. Sci. Technol. 28 $105002,2017$.

[18] B. Dorit, E. Jan, L. Kai and N. Andreas, "The 3D Hough Transform for Plane Detection in Point Clouds: A Review and a new Accumulator Design", 3D Research Center, Kwangwoon University and Springer 2011. 